\title{
Hygroscopic sonographically detectable clips form characteristic breast and lymph node pseudocysts
}

\author{
Moshe Carmon ${ }^{1}$, Sofia Zilber ${ }^{2}$, David Gekhtman ${ }^{3}$, Oded Olsha ${ }^{1}$, Tal Hadar ${ }^{1}$ and \\ Eliahu Golomb ${ }^{2}$
${ }^{1}$ Breast Health Unit, Shaare Zedek Medical Center, Hebrew University, Jerusalem, Israel; ${ }^{2}$ Department of Pathology, Shaare Zedek Medical Center, Hebrew University, Jerusalem, Israel and ${ }^{3}$ Department of Breast Imaging, Shaare Zedek Medical Center, Hebrew University, Jerusalem, Israel

\begin{abstract}
The use of hygroscopic sonographically detectable clips (HSDCs) has dramatically increased during the last years, especially in breast cancer patients who undergo neoadjuvant chemotherapy. The aims of this study are to define the appearance of HSDC sites in histopathological specimens, and to enable pathologists to recognize these sites and differentiate them from other lesions. We examined 124 breast cancer specimens in which the application of HSDCs was documented, 88 breast tissues and 36 lymph nodes, and analyzed the appearance of the clip site in these tissues. The clip site was clearly detected histologically in $79 / 88(90 \%)$ of the breast specimens and in $29 / 36(81 \%)$ of lymph node specimens. In most of the specimens, the HSDC site had a specific characteristic appearance of a pseudocyst, lined by layers of epithelioid histiocytes, sometimes with pseudopapillary formation, and with minimal or no fibrosis. This was the appearance in 69 of the breast specimens and in $\mathbf{2 3}$ of the lymph node specimens. In other specimens, scarring, scattered foamy macrophages and abundant siderophages were the predominant findings, as usually found in sites of other clips. As non-palpable breast lesions become more frequent, clips play a major role in the treatment of breast cancer, making them an important component of the communication among radiologists, surgeons, pathologists, and oncologists. HSDCs in tissues have a characteristic appearance with an epithelioid component. Pathologists should be able to recognize this finding, differentiate it from other breast lesions and include it in the pathology report.
\end{abstract}

Modern Pathology (2018) 31, 62-67; doi:10.1038/modpathol.2017.96; published online 11 August 2017

Increased availability of screening for breast cancer and compliance to screening, as well as improved sensitivity of modern screening tools, have led to a high rate of detection of very small breast tumors. ${ }^{1}$ Such small cancers may be completely removed by percutaneous sampling. ${ }^{2}$ Improved anti-cancer drugs and patient selection for neoadjuvant treatment increase the rate of complete radiological and pathological response of breast cancers. ${ }^{3}$ To prevent loss of tumor location in such instances, marker metal clips are often used in breast cancer surgery over the last two decades. ${ }^{4,5}$ Markers are commonly used for inconspicuous lesions, for tumors planned

Correspondence: Dr M Carmon, MD, Breast Health Unit, Shaare Zedek Medical Center, Hebrew University, 12 Beyth St., POB 3235, Jerusalem 91031, Israel.

E-mail: carmonm@gmail.com

Received 6 May 2017; revised 11 June 2017; accepted 18 June 2017; published online 11 August 2017 for neoadjuvant therapy, or in cases in which the biopsy procedure itself removes the lesion or reduces its visibility. ${ }^{6}$ Such clips may also help identify axillary nodes that had metastases prior to neoadjuvant treatment. This is specifically important in centers that practice sentinel lymph node biopsy for involved nodes following chemotherapy. ${ }^{7-9}$

Ultrasound performed by clinicians is rapidly expanding in many medical fields such as anesthesia for delivering nerve blocks, trauma medicine for quick emergency room evaluation, and in breast cancer surgery. ${ }^{10,11}$ Intraoperative ultrasound in breast conserving surgery has been shown to reduce the rate of re-operations. ${ }^{12-14}$ With this background, a wide variety of ultrasound visible tissue localization marker clips have been designed. The availability of such clips offered sonographic targeting as a faster and less expensive alternative to stereotaxis, with less patient discomfort and without exposure to ionizing radiation. $^{6}$ The use of sonographically 
visible clips in conjunction with intraoperative ultrasound often obviates the need for pre-operative localization procedures. ${ }^{15}$

Many types of sonograpically detectable clips (SDCs) from different manufacturers have become available in the last decade. ${ }^{16} \mathrm{~A}$ thorough comparative analysis of their main features, composition, size, and sonographic visibility, with pictures of the different clips and their sonographic tissue appearance, has been published by Seow et al. ${ }^{6}$ The main types of SDCs include: (1) clips made of metal only (titanium, to avail MRI compatibility): Ultraclip II MR (CR Bard, Tempe, AZ, USA) and CeleroMark (Hologic, Marlborough, MA, USA). (2) clips without metal: with carbon-coated zirconium oxide: BiomarC (Carbon Medical Technologies, St Paul, MN, USA). (3) Clips with a combination of a hygroscopic organic material and metal, in which the fluids accumulated by the hygroscopic substance provide sonographic visibility. Most SDCs in the current practice belong to the latter type. They include SDCs with non-absorbable material, such as Ultraclip II PVA, with polyvinyl alcohol (Bard) and clips with hygroscopic bio-absorbable organic material, including V Mark (Angiotech Pharmaceuticals, Vancouver, BC, Canada); Gel Mark Ultra (stainless steel+gel, Bard ); SecurMark (Hologic), and Hydromark (Mammotome, Cincinnati, OH, USA). The selection of the clip to be used depends on regulatory approval in specific countries, ${ }^{17}$ economic parameters, local service considerations, and the personal preferences and experience of the radiologists and surgeons. In our center we have used the hygroscopic Hydromark clip (Mammotome). According to information from the manufacturer, the global annual use of the hydromark clip exceeds 200000 units. Like most widely used clips, it contains a metal and hygroscopic gel organic component. The metal size of this clip is $\sim 2 \mathrm{~mm}$. We previously found that it is sonographically visible for at least 6 months after placement, ${ }^{9}$ tends not to be displaced with time and enables good cross-modality communication. The degradability of its hygroscopic component may be an additional advantage if the lesion does not have to be removed.

The availability of MRI-compatible metal and hygroscopic SDCs (HSDCs) enables a significant facilitation of cross-modality correlation. Lesions seen by MRI only or mammography only become accessible to removal by the use of intraoperative ultrasound if they contain an SDC. ${ }^{6}$ The presence of SDC can help in the localization by the surgeon, especially in presence of additional, benign findings. ${ }^{14}$ With the increased use of neoadjuvant therapy, and the high rates of complete response to it, clips become the target site of breast and lymph node surgical procedures. It is imperative that the pathologist recognizes and describes the appearance of a clip site in the tissue in this setting.

Tissue response to breast biopsy site marking devices has been described in 2005, and was found to be dependent on the type of devices used. ${ }^{17}$ The aim of the present study is to define and characterize the appearance of HSDC sites in breast and lymph node histopathological specimens. For this purpose, we reviewed our database for cases with documented use of HSDCs between 2014 and 2016 in our Breast Service unit, and analyzed the histopathological specimens of the patients who underwent HSDC placement. We found that HSDC sites tend to show pseudocysts lined by epithelioid histiocytes in breast and lymph node tissue.

\section{Materials and methods}

Institutional Review Board authorization was obtained to perform this study, and informed consent was waived.

To identify pathological specimens with hygroscopic clips, we reviewed the prospective database of our breast surgery service, the surgical reports, and the gross pathology reports of 1260 surgical procedures performed in our breast service between 2014 and 2016. This screening led to the finding of 124 breast cancer surgical specimens in which the introduction of HSDCs was documented: 88 breast tissue specimens and 36 lymph node specimens. The time period between the clip placement and the resection procedure varied from 1 day to 7 months. The sonographically detectable clip used was the HydroMARK clip (Mammotome).

Hematoxylin and eosin (H\&E) stained slides were examined in all cases. The cellular components, degree of inflammation, fibrosis, neovascularization, and presence of birefringent foreign material were analyzed in all cases.

\section{Results}

The histologic detection rate and appearance of HSDCs in breast and lymph node tissue is summarized in Table 1. The clip site was clearly detected

Table 1 Detection and appearance of the sonographically detectable clip Hydromark (Mammotome) in breast and lymph node tissue

\begin{tabular}{|c|c|c|c|c|c|}
\hline Tissue & $\begin{array}{c}\text { Pseudocyst-minimal } \\
\text { or no fibrosis }\end{array}$ & $\begin{array}{l}\text { Pseudocyst- } \\
\text { prominent fibrosis }\end{array}$ & $\begin{array}{l}\text { Foreign body reaction } \\
\text { without pseudocyst }\end{array}$ & $\begin{array}{l}\text { Fibrosis, hemorrhage, and } \\
\text { hemosiderin deposition }\end{array}$ & $\begin{array}{l}\text { Clip site not } \\
\quad \text { found }\end{array}$ \\
\hline Breast & 69 & 4 & 2 & 4 & 9 \\
\hline Lymph node & 23 & 3 & 3 & 7 & 7 \\
\hline
\end{tabular}


histologically in 79/88 (90\%) of the breast specimens and in $29 / 36$ (81\%) of the lymph node specimens. In most of the specimens, the HSDC site had a specific characteristic appearance of a pseudocyst, lined by epithelioid histiocytes, sometimes with pseudopapillary formation (Figure 1), and with minimal or no fibrosis. This was the appearance in 69 of the breast specimens and in 23 of the lymph node specimens. This appearance was different from that usually encountered in specimens with other types of clips and localization wires, in which scarring, scattered foamy macrophages, and abundant siderophages are the predominant findings.

In a specimen taken 1 day after clip placement, no histiocytic reaction was seen (Figure 2). Three weeks after HSDC placement, histiocytes formed a lining of one layer around the clip site (Figure 3). The content of the clip site was described as gelatinous in some of the pathology report's gross descriptions. In some cases, a substance with a basophilic hue resembling myxoid or mucinous material was noted at the clip site, likely reflecting the organic gel material of the clip (Figure 4). This substance should not be mistaken as an indicator of mucinous carcinoma.

There are rare cases in which a differential diagnosis exists between a clip site and viable carcinoma after neoadjuvant chemotherapy. Figure 5a shows a lymph node, 5 months after clip placement in a metastatic tumor, and after neoadjuvant chemotherapy (cyclophosphamide, doxorubicine, and paclitaxel) administered during these months. Two cystic spaces with different linings are seen in the lymph node: One of them, in the upper part of the micrograph, is a pseudocyst formed by the hygroscopic clip. The other cystic formation is lined by viable tumor cells. Immunostains for CD68 and cytokeratin delineates the nature of the cystic spaces (Figure $5 \mathrm{~b}$ and $\mathrm{c}$ ).

The main cellular hallmark of the appearance of clip sites in lymph nodes is epithelioid histiocytes, sometimes forming structures mimicking pseudopapillations, with granulomatous reaction. These features are also found in frozen sections taken intraoperatively as sentinel lymph nodes (Figure 6).

\section{Discussion}

In this work, we show that hygroscopic, sonographically detectable clips elicit a tissue response that can be specifically identified by the pathologist. This response is the formation of a pseudocyst lined by histiocytes.

In the classic view, the process of breast cancer surgery is the excision of palpable breast masses by mastectomy or breast conserving excision. Currently, many surgical procedures are directed at nonpalpable lesions. ${ }^{11,14}$ The technological improvements in mammography, together with the introduction of breast ultrasound and MRI for screening purposes, and the increased public awareness have

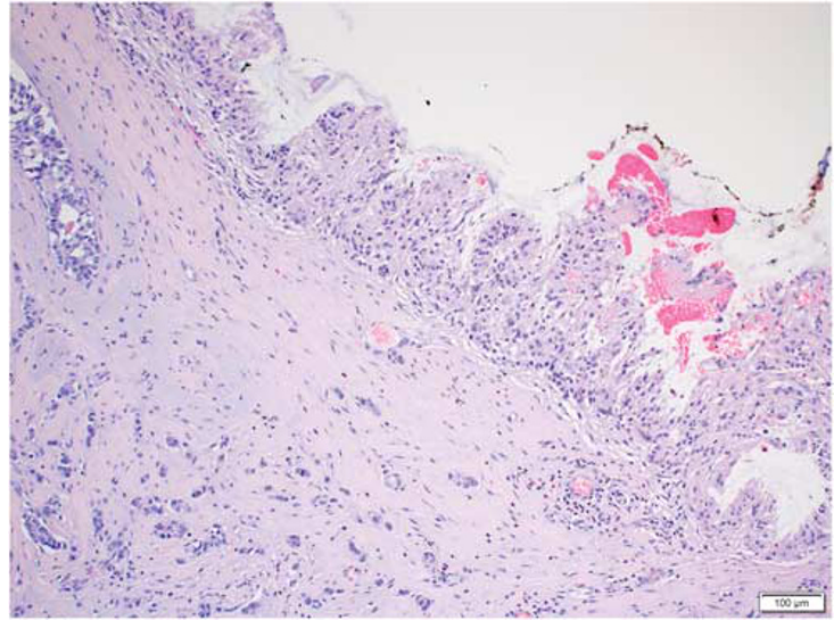

Figure 1 Photomicrograph of clip site within a carcinoma. The specimen was removed 8 weeks after clip placement. The clip induced pseudocyst (right upper side) is lined by histiocytes, forming pseudopapillary projections. Infiltrating carcinoma is seen in the left side. Original magnification $\times 100$, H\&E staining.

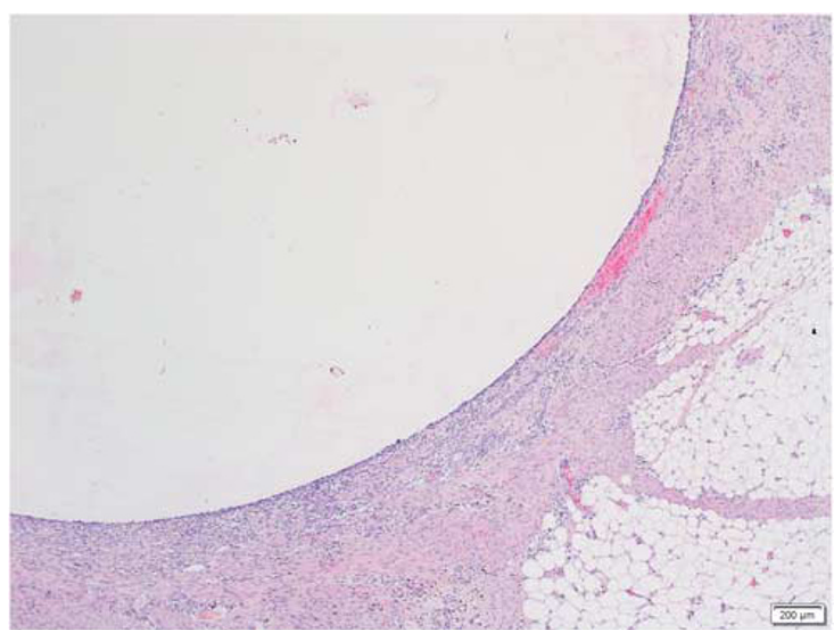

Figure 2 Photomicrograph of clip site one day after clip placement. The clip induced a fluid-filled space in the tissue, still without a lining. Original magnification $\times 40$, H\&E staining.

dramatically improved the ability to identify suspicious non-palpable lesions. Very small lesions are no longer rare and the rate of non-palpable cancers approaches $50 \%$ of patients in many centers and continues to grow. ${ }^{13,18}$ This trend shifts the physical target in breast surgery from the tumor itself to surrogate markers such as localizing wires, radioactive seeds, or metal clips in many operations.

Situations in which clips may constitute the physical target of the surgical procedure include non-palpable cancers, sites of tumors that were removed by percutaneous biopsy procedures, and tumors sites after neoadjuvant chemotherapy, especially in the face of complete radiologic response. In the latter situation, there is a long time gap between the localization of the tumor and the surgical procedure. 


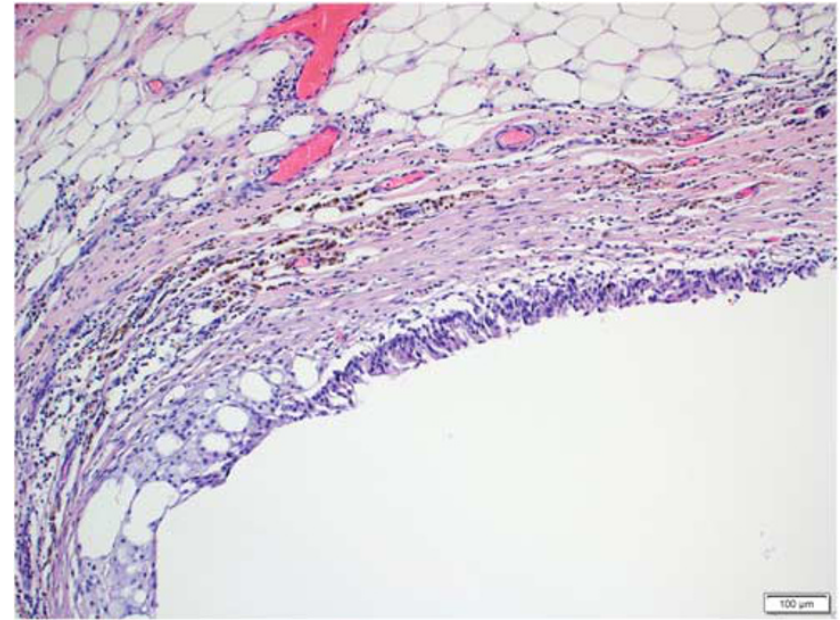

Figure 3 Photomicrograph of clip site 3 weeks after clip placement. A layer of histiocytes lines part of the cystic space induced by the clip. Original magnification $\times 100, H \& E$ staining.

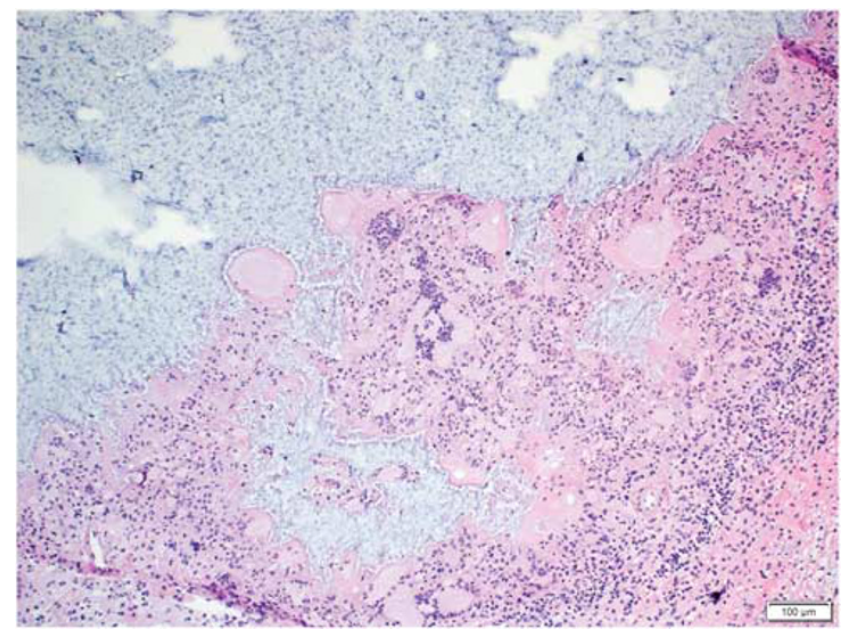

Figure 4 Photomicrograph of a frozen section of a clip site in a lymph node 6 months after clip placement. A substance with a basophilic hue, reminiscent of myxoid or mucinous material accumulated at the clip site. No viable tumor was found in this specimen. Original magnification $\times 100, \mathrm{H} \& \mathrm{E}$ staining.

The aim of the pathologist who analyzes the surgical specimen is to identify every possible pathological process in the specimen. When the lesions are very small, or in the face of a complete or nearly complete response to chemotherapy, the pathologist must recognize the localization markers used by the surgeon not only in the gross specimen, but also at the tissue level. The recognition of a biopsy tract can occasionally have a dramatic impact on the diagnosis and treatment. ${ }^{19}$

The histological appearance of localization marker sites has been characterized for different clips. 17,20 To the best of our knowledge, the specific histologic response to hygroscopic clips has not been addressed.

Most biopsy tracts, wires, and clips elicit a similar histologic response of local inflammation, foreign body reaction, fat necrosis, fibrosis, and pigment
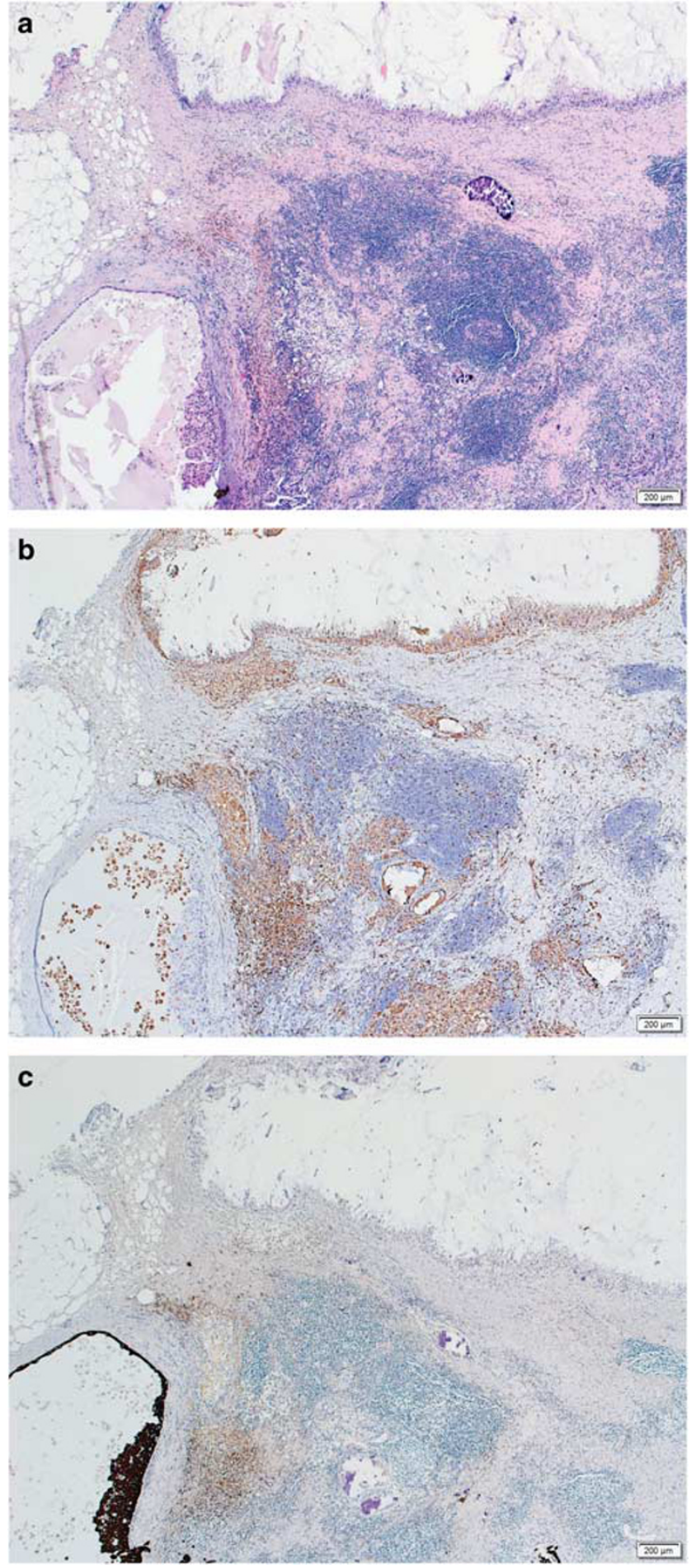

Figure 5 Photomicrograph of a lymph node, 5 months after clip placement. The lymph node was involved with metastatic carcinoma at the time of clip placement. Two cystic spaces are found: the pseudocyst in the upper part of the micrograph is the site of the hygroscopic clip and the cystic space in the left lower part of the micrograph is a tumor that showed partial response to the treatment with cyst formation, lined by viable tumor cells. Original magnification $\times 40$. (a) H\&E staining. (b) Immunostain for CD68. (c) Immunostain for cytokeratin. 


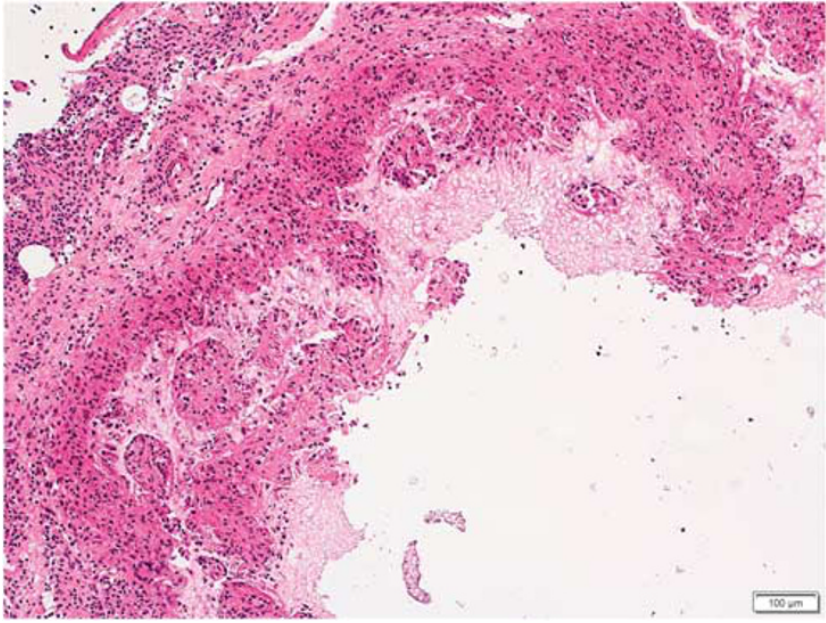

Figure 6 Photomicrograph of a frozen section of a clip site in a lymph node 3 months after clip placement. Prominent granulomatous reaction, epithelioid cells and pseudopapillary projections are seen. Care must be taken not to misinterpret the epithelioid cells as epithelial. Original magnification $\times 100$, H\&E staining.

accumulation resulting from hemosiderin deposition. ${ }^{17,20}$ Specific markers such as collagen plugs have recognizable histologic appearance. ${ }^{17}$ Inflammation, fibrosis, giant cell reaction, and accumulation of fibrinous material have also been described in response to hygroscopic clips with pellets containing polylactic acid/ polyglycolic acid, similar to those used today. ${ }^{17}$ The characteristic pseudocystic finding in sites of hygroscopic clips described here is unique, and may help the pathologist with navigation in the tissue and interpretation of findings. The difference between the description from 2005 and the present one may stem from differences in the clips themselves, in the procedure of their placement, or from the fact that in 2005 clips were maintained in the tissue for relatively short periods up to 6 weeks, whereas nowadays the duration is longer and may reach up to 6 months. ${ }^{9}$

The pathologic examination of a tissue containing a clip may not be considered complete if the site of the clip is not described. For example, if a hygroscopic clip was placed and viewed by the surgeon in a tumor and the pathologist finds a small malignant tumor in the tissue but can not locate the clip near it, consideration should be taken to verify that the very tumor viewed by the surgeon was indeed found in the tissue grossing. Furthermore, if a clip was placed in a metastatic lymph node before neoadjuvant therapy, and 6 months later a sentinel lymph node is free of viable tumor and shows signs of response to therapy, it is the clip site that tells the pathologist and the surgeon that the examined lymph node is the site in which tumor had been found. In other words: Since the physical target of the surgeon is a clip, a pathology report is not complete without a description of the clip site.

The morphological features of a hygroscopic clip site may raise differential diagnoses. The most obvious one is granulomatous lymphadenitis. In specimens of post-neoadjuvant therapy, histiocytes may raise the differential diagnosis of a tumor site with complete response. Moreover, given the epithelioid appearance of histiocytes and the potential formation of pseudopapillary structures, special attention should be given not to misinterpret this finding as an epithelial metastasis. This may specifically potentially happen in frozen sections of low technical quality of adipose lymph nodes.

The use of HSDCs had a dramatic impact on the communication between the surgeon and the radiologist about the navigation in the breast tissue in the surgical position. ${ }^{9,21,22}$ The ability to morphologically detect the clip site at the tissue level introduces the pathologist into this communication and further enables radiologic-pathologic correlation in surgical specimens.

To summarize, hygroscopic sonographically detectable clips elicit a tissue response characterized by pseudocysts lined by epithelioid histiocytes, with foreign body reaction, and often with minimal or no fibrosis or inflammation. The pathologist should recognize this finding, associate it with the presence of a hygroscopic clip, and include it as an integral part of the pathology report.

\section{Acknowledgments}

The authors declare that the findings were obtained lawfully, according to the law of Israel.

\section{Disclosure/conflict of interest}

The authors declare no conflict of interest.

\section{References}

1 Aracava MM, Chojniak R, Souza JA, et al. Identification of occult breast lesions detected by magnetic resonance imaging with targeted ultrasound: a prospective study. Eur J Radiol 2012;83:516-519.

2 Liberman L, Zakowski MF, Avery S, et al. Complete percutaneous excision of infiltrative carcinoma at stereotactic breast biopsy: how can tumor size be assessed? Am J Roentgenol 1999;173:1315-1322.

3 Goorts B, van Nijnatten TJ, de Munck L, et al. Clinical tumor stage is the most important predictor of pathological complete response rate after neoadjuvant chemotherapy in breast cancer patients. Breast Cancer Res Treat 2017;163:83-91.

4 Dash N, Chafin SH, Johnson RR, et al. Usefulness of tissue marker clips in patients undergoing neoadjuvant chemotherapy for breast cancer. Am J Roentgenol 1999;173:911-917.

5 Youn I, Choi SH, Kook SH, et al. Ultrasonographyguided surgical clip placement for tumor localization in patients undergoing neoadjuvant chemotherapy for breast cancer. J Breast Cancer 2015;18:44-49.

6 Seow JSH, Philips M, Taylor D. Sonographic visibility of breast tissue markers: a tissue phantom 
comparison study. Australas J Ultrasound Med 2012;15:149-157.

7 Caudle AS, Yang WT, Mittendorf EA, et al. Selective surgical localization of axillary lymph nodes containing metastases in patients with breast cancer: a prospective feasibility trial. JAMA Surg 2015;150: 137-143.

8 Boughey JC, Ballman KV, Le-Petross HT, et al. Identification and resection of clipped node decreases the false-negative rate of sentinel lymph node surgery in patients presenting with node-positive breast cancer (T0T4, N1N2) who receive neoadjuvant chemotherapy: results from ACOSOG Z1071 (Alliance). Ann Surg 2632016;802-807.

9 Carmon M, Olsha O, Gekhtman D, et al. Detectability of hygroscopic clips used in breast cancer surgery. J Ultrasound Med 2017;36:401-408.

10 Ahmed M, Abdullah N, Cawthorn S, et al. Why should breast surgeons use ultrasound? Breast Cancer Res Treat 2014;145:1-4.

11 Ngo C, Pollet AG, Laperrelle J, et al. Intraoperative ultrasound localization of nonpalpable breast cancers. Ann Surg Oncol 2007;14:2485-2489.

12 Olsha O, Shemesh D, Carmon M, et al. Resection margins in ultrasound guided breast conserving surgery. Ann Surg Oncol 2012;18:447-452.

13 Ramos M, Diaz JC, Ramos T, et al. Ultrasound-guided excision combined with intra-operative assessment of gross macroscopic margins decreases the rate of reoperations for non-palpable invasive breast cancer. Breast 2012;22:520-524.

14 Bossi D, Amadori R, Nebuloni M, et al. Localization of nonpalpable breast lesions with sonographically visible clips: optimizing tailored resection and clear margins. Am J Surg 2015;209:950-958.

15 Eby PR, Calhoun KE, Kurland BF, et al. Preoperative and intraoperative sonographic visibility of collagenbased breast biopsy marker clips. Acad Radiol 2010;17: 340-347.

16 Sakamoto N, Ogawa Y, Tsunoba Y, et al. Evaluation of the sonographic visibility and sonographic appearance of the breast biopsy marker (UltraClip) placed in phantoms and patients. Breast Cancer 2016;24: $585-592$

17 Guarda LA, Tran TA. The pathology of breast biopsy site marking devices. Am J Surg Pathol 2015;29: 814-819.

18 Langhans L, Jensen MB, Talman MM, et al. Reoperation rates in ductal carcinoma in situ versus invasive breast cancer after wire-guided breast-conserving surgery. JAMA Surg 2017;15:378-384.

19 Olsha O, Reinus C, Carmon M. Demonstration of the preoperative biopsy needle tract: an integral part of the surgical specimen pathology report in breast cancer patients. Breast J 2007;13:627-628.

20 Rogers LW. Breast biopsy: a pathologist's perspective on biopsy acquisition techniques and devices with mammographic-pathologic correlation. Semin Breast Dis 2005;8:127-137.

21 Gittleman MA. Single-step ultrasound localization of breast lesions and lumpectomy procedure. Am J Surg 2003;186:386-390.

22 Ramos M, Diez JC, Ramos T, et al. Intraoperative ultrasound in conservative surgery for non-palpable breast cancer after neoadjuvant chemotherapy. Int J Surg 2014;12:572-577. 